\title{
La internacionalización de la educación: cronología mínima de un término pedagógico
}

\section{The internationalization of education: minimum chronology of a pedagogical term}

\author{
MSc Sandra Martínez López ${ }^{1}$ \\ zyanyacu73@gmail.com \\ ORCID: https://orcid.org/0000-0003-0851-5597 \\ PhD, María Magdalena López Rodríguez del Rey² \\ mmrodirguez@ucf.edu.cu \\ ORCID: https://orcid.org/0000-0003-2776-3756 \\ Lic Eliani Vázquez Vasallo ${ }^{3}$ \\ evasallo@ucf.edu.cu \\ ORCID: https://orcid.org/0000-0001-7340-3474
}

Recibido: 1/9/2020, Aceptado: 1/11/2020

\begin{abstract}
RESUMEN
La internacionalización de la educación se ha convertido en un tema a debate en comunidades educativa. Políticos, pedagogos, padres y docentes intentan ver en este término la manera de resolver la inequidad con que se forman niños, adolescente y jóvenes en un mundo cada vez más global. La discusión gana adeptos, pero poco consenso, en como concretar en la práctica pedagógica este propósito y la tendencia a trasferir y conciliar posturas vuelve una y otra vez, la mirada a la experiencia que se lleva a cabo en la Educación superior en el mundo. Interesados en aportar a este debate En este artículo se sistematizan desde el punto de vista histórico las ideas de los autores más reconocidos en el tema con el objetivo de identificar los aspectos teóricos y metodológicos que den respuesta al reto de la globalización, al asumir modelos educativos que puedan superar las perspectivas nacionalistas por unos más centrados en fomentar el compromiso ciudadano mundial.
\end{abstract}

Palabras clave: Internacionalización de la educación superior, Globalización, Ciudadanía mundial, Proceso de integración.

\section{ABSTRACT}

\footnotetext{
${ }^{1}$ Profesora de la Escuela Primaria Dinh Bo Linh, Ciudad de Ho Chi Minh, Vietnam

2 Docente investigadora de la Universidad de Cienfuegos, Cienfuegos, Cuba

${ }^{3}$ Profesora de Pedagogía Facultad de Educacón Universidad de Cienfuegos, Cuba
} 


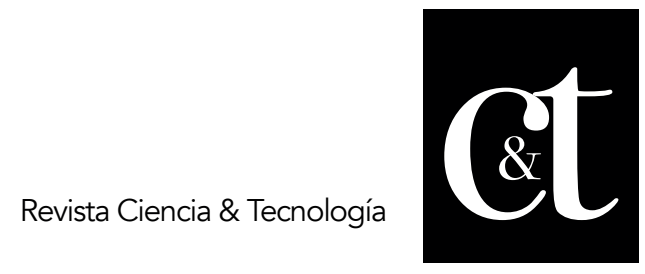

No. 29, 31 de enero de 2021

ISSN impreso: 1390 - 6321

ISSN online: 2661 - 6734

The internationalization of higher education has become a subject of debate by the educational community. Politicians, pedagogues, parents, and teachers try to see in this term the way to resolve the inequity with which children, adolescents and young people are formed in an increasingly global world. The discussion gains followers but little consensus on how to materialize this purpose in pedagogical practice, and the tendency to transfer and reconcile positions returns over and over again, to the experiences carried out in higher education in the world. The purpose of this article is to contribute to this debate. The ideas of the most recognized authors on the subject are systematized from a historical point of view with the aim of identifying the theoretical and methodological aspects that respond to the challenge of globalization, by embracing educational models that can overcome the nationalist perspectives with other options more focused on fostering global citizen engagement.

Keywords: Internationalization of higher education, Globalization, World citizenship, Integration process.

\section{Introducción}

Aunque la referencia a la educación internacional se ha considerado como un instrumento esencial en la concreción de la política exterior de las naciones, sobre todo en lo referente a la seguridad nacional y a la paz, en la actualidad crece la importancia que una vez tuvo en los intercambios culturales, científicos y educativos internacionales, en la formación y desarrollo de la personalidad integral planteada como propósito de la educación en los sistemas educativos nacionales en el mundo.

En efecto durante décadas se viene creando una compleja y amplia red de relaciones para asegurar que los productos y servicios educativos formarán parte sustancial de su política exterior. De hecho, en política exterior se observan cambios importantes: antes se consideraba que la educación contribuyó a valorar la educación como una actividad que podía contribuir al desarrollo un programa cultural exportable y al mismo tiempo un aspecto clave en el propósito de formar a las nuevas generaciones en una perspectiva de reconocimiento, el respeto y disfrute de la cultura, sobre todo, de los países con los que se mantienen relaciones diplomáticas y de colaboración económica.

La internacionalización de la educación es un tema que centra la atención de los educadores y directivos de las instituciones educativas del mundo. Al entender el fenómeno de la globalización y su influencia en el ámbito educativo, los análisis en este tema han emigrado desde una visión sofisticada, privilegio de escenarios universitarios elitistas y privados, hasta su conversión en políticas prioritarias asumidas por las Universidades del mundo. Construir un sistema educativo mundial inclusivo, heterogéneo y que responda a los retos de procesos políticos, sociales y económicos globalizados son las perspectivas en la actualidad.

En principio se reconoce que, desde su aparición en el ámbito pedagógico, se vincula a la Educación superior. Gacel-Ávila (2016) precisa que entre los retos que impone un mundo globalizado, para las autoridades políticas, económicas e intelectuales, resulta esencial comprender la relevancia de lograr una re-convergencia del ámbito educativo universitario. 


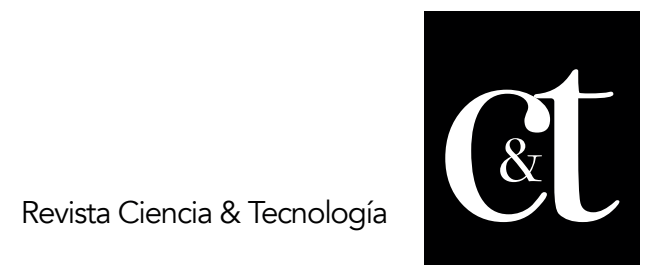

No. 29, 31 de enero de 2021

ISSN impreso: 1390 - 6321

ISSN online: 2661 - 6734

Esto ha llevado a promover convenios y programas de colaboración académica internacionales que garanticen la doble titulación universitaria, la movilidad estudiantil, el posicionamiento, reconocimiento y liderazgo de las universidades a nivel mundial. Sin embargo, en la actualidad el eje fundamental de este proceso se vincula a la formación de un ciudadano del mundo con una visión global y una conciencia integral, en congruencia no sólo, con la realidad sociocultural, económica y política propia, sino con las problemáticas internacionales.

En esta dirección la reflexión y los debates planteados por el (Banco Mundial, 1999; el Consejo Europeo, 2006; UNESCO, 1995; 2015; 2016), están centrando la atención, en superar un marco referencial puramente nacionalista y promover desde políticas educativas de consenso internacional, el propósito de una educación que incluya la ciudadanía mundial en estrecha relación con el desarrollo sostenible.

Las iniciativas y propuestas que han logrado reconocimiento en este tema se hacen eco de diversas posiciones y enfoques, fundamentalmente en lo que respecta al ámbito universitario. Autores como: Knight (1994; 2003; 2018); Delors, (1996); Hans de Wit, $(2001 ; 2010 ; 2011,2013)$ Pedregal $(2003 ; 2018)$. permiten establecer los aspectos que han marcado el proceso a través de la historia y que aportan sin dudas, aspectos que constituyen claves en la transposición pedagógica que es necesaria, para lograr sistemas educativos nacionales que sustenten la internacionalización en la organización del proceso formativo.

En correspondencia con esta posición, en los últimas 40 años se viene apreciando un cambio en la concepción pedagógica, para contribuir a la internacionalización de la educación. En razón de considerar las posibilidades, de que ésta se convierta en una alternativa viable, para ampliar la dimensión formativa y cultural de las nuevas generaciones, ante la influencia de procesos como: la globalización, el desarrollo tecnológico, el acceso inmediato a la información, las nuevas construcciones del conocimiento, desde un enfoque proactivo.

De este modo, se alzan voces y se convoca a estimular la internacionalización como una manera viable de contribuir a concretar competencias globales/internacionales. Los sistemas educativos nacionales, orientados a desarrollar nuevos saberes, actitudes y valores de carácter sociocultural enfocados al desarrollo de ciudadanos con una visión local, nacional y global. Se pretende así que toda vez que sean capaces de convertirse en miembros productivos, innovadores y responsables que se implican en la preservación y promoción de la cultura nacional. Al mismo tiempo se enfatiza en el respeto a la diversidad cultural, contrarrestando el efecto homogeneizador que se percibe con la globalización.

Pero, para entender los procesos de internacionalización en educación debe hacerse distinción importante de su alcance; pues, no se trata de verla como un fin en sí mismo, sino como una manera de contribuir a la formación de ciudadanos capaces de servir a los intereses nacionales y particulares de cada región en función de la calidad de la educación. Toda vez, que se proporcione una vinculación con la vida y la cultura mundial y se promuevan espacios para satisfacer necesidades nacionales. 


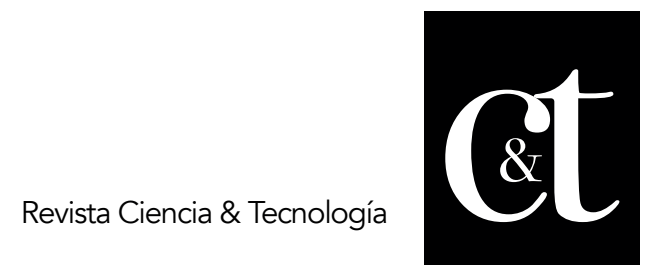

No. 29, 31 de enero de 2021

ISSN impreso: 1390 - 6321

ISSN online: 2661 - 6734

El estudio que se presenta devela los giros y sinergias que caracterizan el proceso.

\section{Materiales y Métodos}

El estudio incluyó la revisión de artículo, tesis, congresos, conferencias y documentos normativos, pero exigió de las autoras establecer requisitos metodológicos esenciales en este proceso. Se optó por la sistematización de los estudios aquí referenciados desde una perspectiva histórica que apunta significar los tiempo y espacios de reflexión desde los que emergen, sirvieron de base para develar el compartiendo de las ideas que sustentan las definiciones y caracterización de las concepciones acerca del término y de los rasgos que caracterizan a este proceso y el alcance que se evidencia en su evolución, hasta su conversión en un aspecto esencial en la concepción pedagógica de la educación en la actualidad.

Como exponentes de referencia para el marco teórico de esta investigación, las autoras asumen los aportes realizados por autores como:

- Hans de Wit (Holanda) quien, además de establecer un recorrido histórico sobre el tema, analiza y resalta los diferentes aportes recogidos en los modelos de internacionalización acotados por Neave (1992); Rudzki (1993); Davies (1992); Van Dijk, (1995); Van der Wende (1997) y el de Knight (1994).

- Van der Wender (Holanda) diseña un modelo conceptual sobre la internacionalización como proceso con énfasis en el análisis del contexto, la implementación y los efectos a largo plazo desde la institución educativa y en estrecha relación con las políticas nacionales y sus objetivos y metas

- Jane Knight (Canadá) es, desde la mirada de este estudio, la autora que realiza una definición más integral en su modelo de internacionalización de la educación superior al comprender su enfoque de proceso transformador integral desde una dimensión internacional e intercultural donde son inseparables identidad y cultura.

- Jocelyne Gacel-Ávila (México) establece reflexiones, que han sido asumidas en este artículo, sobre la repercusión de la internacionalización en el desempeño de los estudiantes y el amplio abanico de posibilidades que ofrece en el intercambio cultural asumiendo la globalización como reto para mantener y ampliar el alcance de las identidades.

Desde estos aportes, el estudio realizado para este artículo, sobre el proceso de internacionalización de la educación, argumenta criterios como:

- El análisis histórico de las iniciativas, posiciones o enfoques y su evolución,

- Los denominados sectores y grupos involucrados.

- Los riesgos en que se pueden incurrir toda vez que se implemente dicho proceso como alternativa pedagógica en la era de la globalización.

\section{Resultados y Discusión}

Al rastrear en artículos, ensayos, se advierte la posición que varios autores (Benéitez, 2004; Heater, 2007; González, 2011; Casado, 2016; Sarochar, 2018) han dejado planteada al referir, que este proceso tiene sus orígenes en las Universidades Europeas de la edad media; su carácter universal y las relaciones internacionales constituyen un hecho irrefutable.

En este caso, el acto de otorgar una licencia a los docentes que le confería el derecho a ejercer dentro de la propia institución y en el resto de los centros pertenecientes 29 


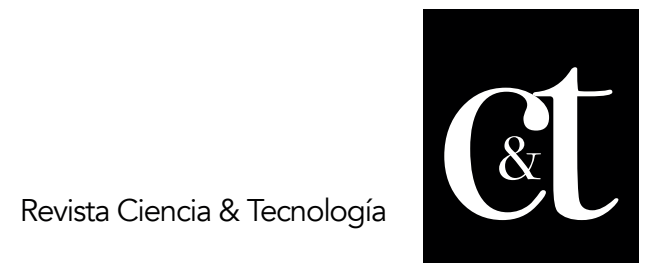

No. 29, 31 de enero de 2021

ISSN impreso: 1390 - 6321

ISSN online: 2661 - 6734

al ámbito cultural universitario europeo y que el principal propósito fuera transferir conocimientos religiosos y filosóficos solidos a sus estudiantes, dan sustento a esta aseveración.

En este mismo sentido, las concepciones de la época avalan un único currículo, con la facilidad de revalidación de los estudios y un idioma común, que sentó las bases para una universidad única, independientemente de cual fuera el país donde se ubicaba.

El análisis histórico de las iniciativas, posiciones o enfoques que asumen las universidades muestra después, el devenir de centros más nacionalistas y regionalistas con divergentes modelos a partir de la división del mundo católico y protestante, acentuándose el sentimiento de estado nación en la época de la revolución industrial.

El creciente interés en la internacionalización de la educación, defendida desde las universidades, llegó a alcanzar cierta novedad y le adjudicó alguna relevancia al término. Sin embargo, en un primer momento, la notoriedad conceptual metodológica se le reconoce, a finales de los 80 , a la creación de la Comunidad Económica Europea, en el surgimiento de la necesidad de fomentar un ciudadano más allá de los límites de los estados naciones y con un pensamiento más regional, considerando como pieza clave de este propósito una Educación con este alcance.

En efecto, la evolución de estas ideas permite declarar al menos cuatro aproximaciones a la internacionalización de la educación en la literatura. Expuestas por Qiang (2003: p.3) que cita, por sus similitudes de enfoque, los estudios aportados por: (Arum y Van de Water, 1992; De Wit, 1995; Knight; 1994; 1996; 1997), en un primer momento se aborda la denominada: perspectiva de la actividad, que consiste en la promoción de actividades tales como: intercambio curricular y de estudiantes y docentes, asistencia técnica, y estudiantes internacionales, sobre todo en la educación superior y técnica. Esta perspectiva ha sido muy popular; no obstante, puede ser un acercamiento reduccionista y fragmentado de la educación internacional cuando las actividades son programadas sin tener en cuenta los contextos políticos, sociales y culturales en los que las instituciones está inmersa.

La segunda es la perspectiva de competencias, que hace énfasis en el desarrollo de conocimientos, habilidades, actitudes y valores que se orientan a la búsqueda, generación y transferencia del conocimiento como base para la formación y desarrollo de competencias interculturales. Mientras, la tercera perspectiva es la perspectiva del ethos, que enfatiza la creación de una cultura o clima que valore y apoye las perspectivas e iniciativas internacionales/interculturales. Este enfoque implica considerar la internacionalización mucho más allá de la movilidad o del uso de una segunda lengua para generar un cambio en las prácticas culturales institucionales.

Asimismo, la cuarta perspectiva del proceso, que hace énfasis en la integración de la dimensión internacional en la enseñanza, la investigación y el servicio a través de la combinación de diferentes actividades, políticas y procedimientos que forman parte de un proceso. Sin embargo, desde los estudios de la internacionalización de la educación superior Qiang, (2003), incluye también una perspectiva centrada en quienes la realizan. 


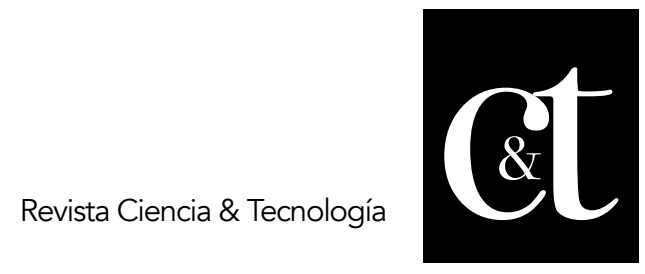

No. 29, 31 de enero de 2021

ISSN impreso: 1390 - 6321

ISSN online: 2661 - 6734

Al respecto se identifican tres sectores principales. Son denominados sectores y grupos involucrados, cada uno con un punto de vista individual, y quizá diferente, acerca de por qué (y cómo) debe internacionalizarse la educación Los tres grupos son: el sector gubernamental, el sector educativo y el sector privado.

El sector gubernamental incluye a los distintos niveles de gobierno que abarcan desde los órganos supranacionales hasta los nacionales, regionales y locales. A este sector pertenecen grupos que tienen intereses gubernamentales, relaciones exteriores, cultura, desarrollo económico y comercio, ciencia y tecnología; pero que también se interesan por dejar la impronta de la internacionalización como una dimensión de la educación.

El sector educativo es igualmente diverso porque es necesario considerarlo desde los niveles de sistema, institucional e individual. Entre los distintos grupos se incluyen los diferentes tipos de instituciones (universidades, politécnicos e institutos) que conforman el sistema; los grupos de investigación y disciplina profesional; las asociaciones de profesionales; los estudiantes, maestros e investigadores; los administradores $y$, por supuesto, otros grupos sectoriales.

El sector privado es otro grupo heterogéneo en virtud de los variados intereses de las empresas de los sectores industrial, comercial y de servicios, la naturaleza de sus productos y servicios, así como sus intereses geográficos. Otro factor de influencia es el tamaño de la empresa y si es local, nacional o transnacional. Es esencial reconocer que el sector privado es mucho más amplio que los proveedores de educación particular.

Así, al coincidir con de wit, (2001), la educación internacional en la universidad debe ser concebida no sólo el currículo, intercambios internacionales de estudiantes en programas cooperativos con la comunidad, entrenamiento y formación de los servicios administrativos, sino que también debe considerarse como un compromiso distinto, actitudes y de conciencia con una orientación global, que trascienda por completo la institución y los marcos de ethos.

A inicios de los 90, Arum \& Van de Water, (1992) al tomar como base los trabajos de Harari, establecen un enfoque tripartito, claves que pueden actuar de manera independiente $o$ integrada en proyectos orientados a la internacionalización de la educación. En este propósito vaticinaron la jerarquía que se le otorgaría a las distintas decisiones que debían ser analizados.

En este sentido, el primer elemento al que se le concede importancia es a la internacionalización de los contenidos del currículo, lo que implica agregar materias y dedicar atención al estudio de los idiomas fundamentalmente.

El segundo aspecto clave, se le atribuye a la Movilidad internacional, referida tanto a los estudiantes como a lo relacionado con entrenamientos e investigaciones, para de esta forma asegurar un ejercicio de reciprocidad académica, fortalecer las relaciones entre universidades y ampliar la experiencia intercultural.

En un tercer elemento, se subraya la asistencia técnica internacional y la concreción de programas cooperativos desde los cuales los avances del desarrollo académico e investigativo podrían convertirse no sólo en una oportunidad de intercambio sino de búsqueda conjunta de vías para el desarrollo.

Sin embargo, la internacionalización de la educación como proceso integrador encuentra su referencia más significativa en la obra de Knight (1993), al definir la internacionalización de la educación superior como el proceso de integración de la 


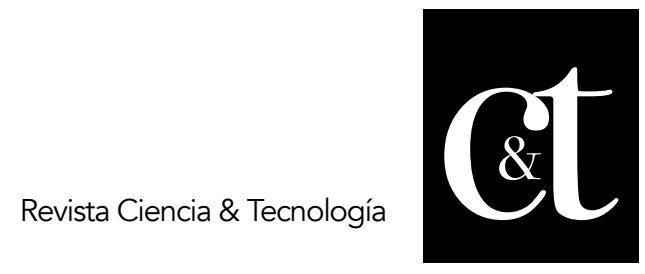

No. 29, 31 de enero de 2021

ISSN impreso: 1390 - 6321

ISSN online: 2661 - 6734

dimensión internacional en la enseñanza, la investigación, servicios y funciones deuna institución de educación superior. Esta comprensión como proceso confiere al término un carácter continuo de formación y desarrollo, implica a las universidades y sus políticas educativas, pero deja fuera de su alcance al menos explícitamente la necesidad de una educación ciudadana integral que responda a los retos que en la actualidad enfrenta un mundo cada vez más globalizado.

A partir de estas ideas Hans de Wit (1993) hace énfasis en el enfoque de proceso que asume la internacionalización, y en la perspectiva global y neutral que se debe tomar. No hace distinción entre Internacionalización y Educación Internacional para él son procesos mediante el cual la educación es desarrollada hacia una mayor dirección con impactos en el flujo de nuevos conocimientos, de académicos, de estudiantes y el contenido del currículo.

Estas ideas, sistematizadas por Knight (1994), insisten en que la internacionalización de la educación superior debe ser entendida como el proceso de integración en una dimensión de alcance internacional en la enseñanza aprendizaje, la investigación y los servicios y funciones universitarias.

Desde una perspectiva de actividad o servicio que introduce o integra enfoques internacionales, interculturales y visiones globales en la directriz principal de una institución de educación superior. Aunque en estos años se comenzó a gestar la necesidad de concebir una educación que trascienda lo particular y le confiera una connotación humanista a la definición, todavía no abarca en su totalidad la verdadera dimensión de la internacionalización como vía para el fomento de una educación ciudadana mundial.

Con un alcance declarado, de lograr la excelencia universitaria, para responder a los retos de un mundo globalizado, una economía exigente y un mercado altamente competitivo autores como: Van der Wende, (1996); Mestenhauser \& Ellingboe, (1998) y Rudzki, (1998) proyectan la internacionalización como generadora de un proceso de cambio organizacional, de innovación curricular, del perfil internacional de los académicos y del personal administrativo, así como la movilidad de los estudiantes. Pensar en el diseño de un contexto educativo diferente en su dimensión internacional que involucre a todos los actores que intervienen en el proceso de enseñanza aprendizaje con la finalidad de lograr calidad en la docencia, la investigación y en todas las actividades que son parte de las funciones universitarias es un proceso proactivo en la búsqueda de una formación integradora y de vanguardia.

Entrando el siglo XXI, el debate en torno a las problemáticas_mundiales de la educación llevó a De Wit, (2001) a establecer, junto a autores estadounidenses, otra acepción: Educación Internacional y hacen énfasis en las actividades, competencias y el ethos, mientras los autores Europa, Canadá y Australia tienden a ampliar el alcance de la internacionalización de la Educación, para reiterar el carácter de proceso integrador e intercultural que deben tener los procesos de enseñanza, de investigación, así como de los servicios y funciones de las instituciones universitarias. 


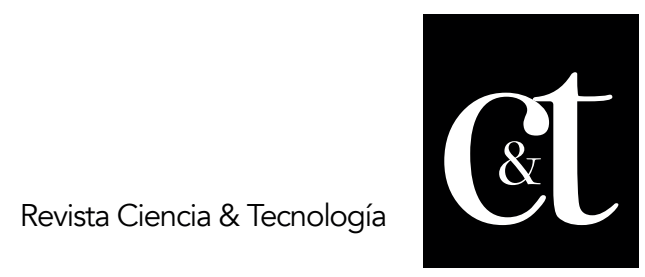

No. 29, 31 de enero de 2021

ISSN impreso: 1390 - 6321

ISSN online: 2661 - 6734

En este sentido Pedregal, (2003) asume que la internacionalización debe verse como una relación bidireccional se da entre las instituciones educativas de los países desde el cual es preciso centrar los procesos educativos en la persona, en las relaciones entre individuos, en los grupos y en las naciones. A través de la socialización, el educando comprende al otro y al mundo para empezar a comprenderse a sí mismo con responsabilidad, solidaridad y aceptación de la diversidad.

Luego, en los trabajos de Qiang, (2003), se pueden identificar al menos cuatro perspectivas de internacionalización de la educación:

- La primera es denominada la perspectiva de la actividad, que consiste en la de actividades tales como intercambio curricular y de estudiantes y docentes, asistencia técnica, y estudiantes internacionales, sobre todo en la educación superior y técnica. Esta perspectiva ha sido muy popular; no obstante, puede ser un acercamiento reduccionista y fragmentado de la educación internacional cuando las actividades son programadas sin tener en cuenta los contextos políticos, sociales y culturales en los que las instituciones está inmersa.

- La segunda es la perspectiva de competencias, que hace énfasis en el desarrollo de conocimientos, habilidades, actitudes y valores que se orientan a la búsqueda, generación y transferencia del conocimiento como base para la formación y desarrollo de competencias interculturales.

- La tercera perspectiva es la perspectiva del ethos, que "enfatiza la creación de una cultura o clima que valore y apoye las perspectivas e iniciativas internacionales/interculturales" Este enfoque implica considerar la internacionalización mucho más allá de la movilidad o del uso de una segunda lengua, para generar un cambio en las prácticas culturales institucionales.

- La cuarta perspectiva del proceso, que hace énfasis en la integración de la dimensión internacional en la enseñanza, la investigación y el servicio a través de la combinación de diferentes actividades, políticas y procedimientos que forman parte de un proceso.

En este sentido, (Knight, 2003) re-define la internacionalización de la Educación superior, mantiene su carácter como proceso de integrar una dimensión internacional, intercultural o global en el propósito, las funciones y el producto de la educación universitaria, pero declara la necesidad de involucrar a los niveles nacionales, sectoriales y a las instituciones en este propósito. Más tarde, complementa la definición otorgándole a un carácter transformador de la educación, sincronizando las instituciones en una dinámica global capaz de armonizar lo local, lo regional y lo mundial. Esta autora, analiza la internacionalización como una estrategia de carácter transversal que permite la transferencia de saberes y recursos, para dar respuestas pertinentes y de calidad a las exigencias locales y regionales desde una visión global.

Pese a esta postura (Didou, 2005), se detiene en el análisis de las posiciones políticas que concretan la internacionalización de la educación en América Latina. Al respecto es preciso considerar que hasta la década del 90, la internacionalización fue uno de los temas ausentes en la literatura sobre la educación superior en Latinoamérica y sólo era mencionada, por las asociaciones de universidades con vocación continental o macro regional. 


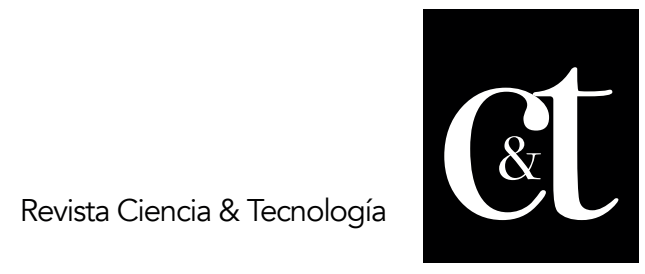

No. 29, 31 de enero de 2021

ISSN impreso: 1390 - 6321

ISSN online: 2661 - 6734

En casi toda la región las autoridades gubernamentales e institucionales comenzaron a inscribir la internacionalización de la educación superior entre sus prioridades: el fin era responder a la globalización y más precisamente, a acuerdos de integración regional en los primeros años del siglo XXI, donde, aunque incipiente; es posible identificar tres modos de concreción.

- La internacionalización endógena y multimodal, que es gestada a partir de la voluntad política de la región de establecer proyectos de cooperación

- gubernamentales o instituciones para hacer frente a problemas y debatir temas de interés común

- La internacionalización macro regional, la cual, basada en la participación de las instituciones de educación superior establecen programas, construyen redes para generar proyectos que deben ir en sintonía con las intenciones de los patrocinadores. - La internacionalización interregional, que se sustenta en los intereses macro regionales, para establecen acuerdos y acciones que deben ser asumidos por los países miembro,

Aunque loables estos modos de concebir la internacionalización de la educación no visibilizan al alumnado que según a Gacel-Ávila (2006), debe ser asumido como el principal beneficiario al considerar que todo cuanto se haga en este propósito debe asegurar que los estudiantes competencias integradoras que responde a los retos individuales y locales, pero desde una perspectiva mundial que les sean de utilidad en su inserción al mundo laboral.

En este caso se asume también que la formación en las instituciones de Educación superior, deben ser concebidas como un proceso que integra en sus funciones sustantivas una dimensión global, internacional, intercultural, comparada e interdisciplinaria que tiene su alcance en el fomento de una perspectiva y consciencia global de las problemáticas humanas en pro de los valores y actitudes de una ciudadanía mundial responsable, humanista y solidaria.

Pero, Knight y UNESCO, (2006) prefieren asumir este término como una dimensión que supone un compromiso a través del cual, la acción de infundir la perspectiva internacional y comparativa de la enseñanza, investigación y servicio de la Educación Superior configura una ética y valores institucionales, que exige ser acogida por el liderazgo institucional, la gobernabilidad, los profesores, los estudiantes, y todas las unidades de servicio y de apoyo académico. En este sentido, Schuerholz-Lehr, Caws, Van Gyn, \& Preece, (2007) precisa que de lo que se trata es de comprender el alcance de este propósito el cual se define en un objetivo loable: prepara para la vida en sociedades plurales por medio de desarrollo de competencia intercultural y una mentalidad global.

Al mismo tiempo, como argumento de pertinencia, Altbach \& Knight, (2007) y Corti, Oliva, y de la Cruz, (2015), enfatizan en la idea de que la internacionalización también aporta al aseguramiento de la calidad y a la innovación de las instituciones académicas, pues al tomar un matiz de solidaridad globalizada en sus proyectos, de los saberes y recursos que se traspasan con el intercambio se deja, como valor agregado, el posicionamiento y la visibilidad institucional Cañón, (2009), considera entonces que la política de internacionalización de la educación deberá ajustarse a las necesidades de cada país y a los intereses de las instituciones académicas, pero sobre todo deberá encontrar los mecanismos que les permitan insertarse de manera 


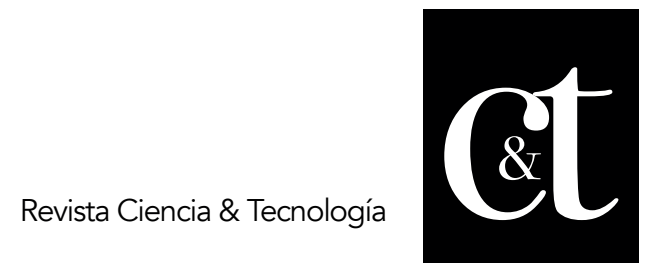

No. 29, 31 de enero de 2021

ISSN impreso: 1390 - 6321

ISSN online: 2661 - 6734

activa en la sociedad global sin dejar de lado la responsabilidad social mejorar su calidad académica y la manera de superar la inequidad existente entre los sistemas educativos.

Según Uribe (2011), la institucionalización de la Educación superior, hacen parte ya del lenguaje y las preocupaciones diarias de los lideres académicos la búsqueda de respuestas para la planificación y ejecución de actividades curriculares, que permitan una formación integral dentro de un marco internacional para el crecimiento económico sostenible en un ambiente de justicia social donde se promueva el desarrollo de la solidaridad, la conciencia o responsabilidad y el compromiso.

Entonces, como señala Cambours de Donini, (2011), es posible reconocer una relación entre el establecimiento de los bloques regionales de cooperación económica, como es el caso de la Unión Europea, MERCOSUR y NAFTA, y los procesos de internacionalización de la educación. Cada país busca encontrar su lugar en la sociedad globalizada y los representantes de los países que conforman estos bloques, comienzan a definir propuestas de metas comunes y acuerdos que acentúan las relaciones interuniversitarias para dar cobertura a las estrategias de movilidad de estudiantes y docentes, a la investigación interinstitucional, la convalidación de títulos, la definición de criterios de evaluación y transferencia de créditos académicos, lo que luego, se concreta en la internacionalización del currículo, los programas de asistencia, la inserción de temas regionales, el aprendizaje de idiomas, la capacitación intercultural, la utilización de las tecnologías de la información y la comunicación, en propuestas formativas presenciales y virtuales.

Al hilo de estos análisis estos autores, también señalan los riesgos que se pueden incurrir al ejecutar las diversas estrategias de internacionalización. En atención a este particular se insiste en que estos procesos pueden estimular la pérdida de la identidad cultural el "elitismo", así como la ausencia de una reflexión sobre el rol de la educación superior al asumir cierta tendencia a la homogenización de competencias profesionales que demanda el mercado laboral en el mundo. La predisposición que este proceso a otorgar mayor valoración a las ciencias aplicadas en detrimento de las disciplinas de las ciencias sociales y humanísticas.

En este caso Hudzik, (2011) y Knight, (2012) reconocen que aun cuando en este momento la internacionalización de la Educación Superior se convierte en un imperativo institucional es preciso comprender que, no sólo se trata de la integración de las perspectivas internacional, global, intercultural y comparativa de contenidos en los programas, o inclusión de esta perspectiva en el proceso de enseñanza/aprendizaje sino de reforzar la idea de que este cambio debe ser integral, por tanto su impacto debe ser a todos en la ámbitos de la vida universitaria, dentro y fuera de marcos de la institución.

En este marco Brewer y Leask, (2012) insisten en que está claro que la concreción del proceso de internacionalización de la educación se concreta en la concepción de un currículo, con orientación internacional del contenido y/o de las formas de preparación a los estudiantes para conducirse en un mundo interdependiente y desigual pero que en cualquier caso de lo que se trata es de contribuir a remediar las inequidades formativa que limitan el desempeño (profesional y socialmente) estudiantes y profesionales en un contexto internacional y multicultural cada vez más global. 


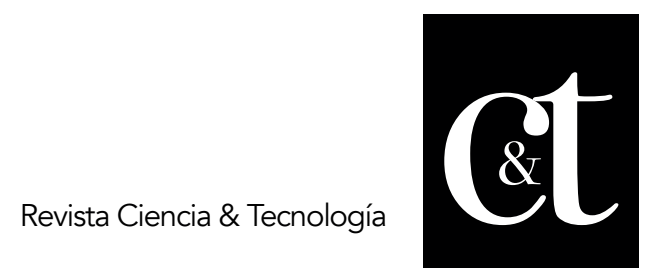

No. 29, 31 de enero de 2021

ISSN impreso: 1390 - 6321

ISSN online: 2661 - 6734

En este empeño Voogt y Pareja ,(2012) comparten la idea de que la internacionalización de la educación aboga por un proceso formativo incluyente y comprometido con la solución de problemas globales $y$, por tanto, enfocada a estándares internacionales de calidad y pertinencia, a la luz de las más apremiantes problemáticas humanas de orden global.

Sin embargo, al compartir la idea de (Hernández y Amador, 2016), es preciso asumir que esta perspectiva sustenta la necesidad de humanizar, de construir ambientes de paz, de formar conciencia ciudadana y que, para ello es preciso crear las condiciones para desarrollar una conciencia social y una cultural centrada en la formación de una Ciudadanía global y el Entendimiento de su lugar en el mundo. Luego, la internacionalización de la educación (superior) se describe como "el proceso de integrar una dimensión global e intercultural a los objetivos, contenido y formas de enseñanza/aprendizaje, a la investigación y todas las actividades y servicio de una institución o del sistema educativo, parta poner de relieve la relación entre naciones, personas, culturas, instituciones y sistemas, y entre naciones convirtiéndose en un dispositivo catalizador, para enfrentar los retos de la globalización vigente.

Al centrar la internacionalización sobre las capacidades, se entiende entonces que los cambios inherentes a la formación de los estudiantes deben partir desde los modelos pedagógicos y educativos institucionales que a nivel meso, macro y micro currículos, estén actualizados e integrados a las necesidades del entorno, tanto propios de un país como a los de la región y el mundo. En virtud de ello, los estudiantes egresan preparados para asumir su contribución al desarrollo científico tecnológico y a la transformación social, al tiempo que se promover interacciones y dispositivos organizacionales innovadores con base en saberes acumulados y transferibles en la región y fuera de ella, justificar prioridades y detectar las contrapartes idóneas Didou, (2017).

Luego, la sistematización cronológica de las ideas que se presentan aquí permite develar, no sólo, la evolución del término, sino advertir su relación con la responsabilidad social de la Educación Superior para ofrecer una respuesta coherente con los desafíos de la sociedad global.

El énfasis en su proyección transita desde estrategias para alcanzar la calidad educativa, al insertar la colaboración y el intercambio en la mejora de infraestructura, hasta el acceso al conocimiento científico técnico y el aumento de la capacidad institucional. Esto visto como valor agregado, no debe perder de vista la atención que requiere el proceso formativo, y la influencia que ejercen las experiencias que desarrollan docentes y estudiantes en el encuentro con otras culturas y otros modelos educativos, cuando participan en las redes académicas e investigativas o fomentan el trabajo colaborativo y su visibilidad internacional.

Por tanto, más allá de la posición que se asume, una perspectiva integral de las posibilidades que ofrece la internacionalización de la educación obliga a pensar en la necesidad de encarar nuevas reflexiones, ahora orientadas a enfrentar los desafíos 


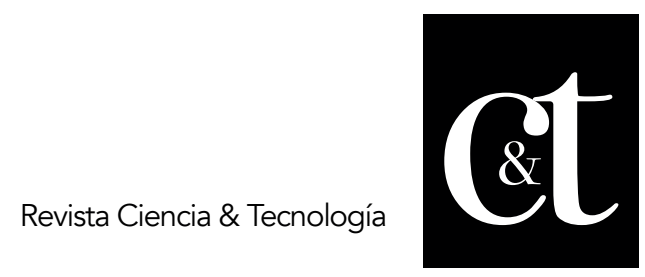

No. 29, 31 de enero de 2021

ISSN impreso: 1390 - 6321

ISSN online: 2661 - 6734

que implican, la trasferencia de estas experiencias a los sistemas de educación general, donde los proyectos e iniciativas aún son limitados y se centran en propuestas de enseñanza de otros idiomas o una visión geopolítica del mundo.

\section{Conclusiones}

La Internacionalización de la Educación Superior se considera una dimensión que asume el modelo pedagógico universitario para responder de manera innovadora a la dinámica del mundo global; por tanto sitúa sus propósitos en afirmar la necesidad de acercar la enseñanza, investigación y servicios a los estándares internacionales desde los que es posible concretar la calidad de la educación y contribuir a formar ciudadanos con una visión global capaces de implicarse de manera responsable en el enfrentamiento de problemas mundiales desde los límites de su localidad o región. El estudio de las diferentes perspectivas de la internacionalización de la educación superior advierte aspectos de su condición de proceso y que debe ser respaldado por las políticas gubernamentales y acuerdos internacionales al asegurar el intercambio justo de conocimientos y recursos, desde la equidad, la solidaridad y la cooperación en virtud de dos aspectos claves: la mejora de la calidad y pertinencia de las universidades y su contribución a la formación de ciudadanos, capaces de asumir con resiliencia la dinámica que impone la globalización.

Los esfuerzos por privilegiar la educación superior como estrategia de internacionalización descansan, no sólo, en la concreción de proyectos que estimulen la movilidad estudiantil, la validación internacional del conocimiento científico tecnológico y las competencias para la vida, sino que deben ser además estimuladas como una alternativa en los marcos de la educación por la paz, la educación para la ciudadanía mundial, el desarrollo sostenible y las relaciones interinstitucionales; objetivos que reclaman organizaciones internacionales y regionales para enfrentar la problemática humanas y social que vive el mundo hoy.

La identificación de la internacionalización de la educación como un término pedagógico queda legitimada en la utilización de esta como objetivo, contenido, forma y tipo de actividad educativa que cobra sentido tanto en la estructura y organización de la institución como en el currículo; pero, deberá además identificarse como un tipo de vivencias y experiencias necesaria para la interpretación de un fenómeno. Tal situación ratifica la imposibilidad de reflejar la realidad a través de nuestro pensamiento, siempre quedarán sistemas por descubrir e interdependencias por señalar, lo que hace del estudio de cualquier fenómeno una tarea inacabada.

\section{Referencias}

Altbach , P., \& Knight, J. (2007). The Internationalization of Higher Education: Motivations and Realities. Journal of Studies in International Education., 11, pp. 290-305.

Arum, S., \& Van de Water, J. (1992). The Need for a Definition of International Education in US Universities, $V$ Bridges to the Future: strategies for internationalizing higher education. . Carbondale: Association of International Education Administrators. 


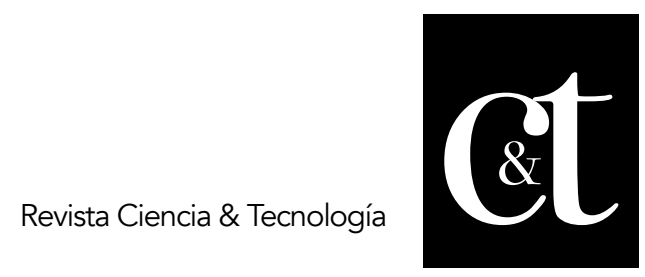

No. 29, 31 de enero de 2021

ISSN impreso: 1390 - 6321

ISSN online: 2661 - 6734

Benéitez, M. (2004). La ciudadanía en la teoría política contemporánea: Modelos propuestos y su debate. Madrid: Universidad Complutense de Madrid. https://core.ac.uk/download/pdf/19709806.pdf

Brewer, E., \& Leask, B. (2012). Internationalization of the curriculum. doi: $10.4135 / 9781452218397 . n 14$.

Cambours de Donini, A. (2011). Internacionalización e integración de los sistemas de educación superior en el Mercosur educativo. , 4(1), Revista de la asociación de sociología de la educación, 4,(1), pp. 59-72. Retrieved from https://ojs.uv.es/index.php/RASE/article/view/8646/8189

Cañón, J. (2009). Internacionalización del currículo: Contexto, implicaciones y conceptos. Seminario sobre internacionalización del currículo - memorias y reflexiones, pp. 87-112.

Casado, D. (2016). Historia y teoría de la educación. España: Centro de Estudios Financiero.

Corti, A., Oliva, D., \& de la Cruz, S. (2015). La internacionalización y el mercado universitario. Revista de la Educación Superior, XLIV (2); No. 174, pp. 47-60. https://reader.elsevier.com/reader/sd/pii/S0185276015000886?token=D16912 5EFF436611657F31A9ED5C3E16F3058D2486D53385B1E50CF7CB6A86CDBEAA 8757098C09F1495509C260A8A168

De Wit, H. (1993). On the Definition of International Education. European Association for International Education Newsletter, no. 11, pp. 7-10. - (2001). Internationalisation of Higher Education in the United States of America and Europe. Amsterdam: in eigen beheer.

. (2010). Internationalisation of Higher Education in Europe and its assessment, trends and issues. Nederlands: Nederlands-Vlaamse Accreditatieorganisatie.

. (2011). Globalización e internaciionalización de la educación superior. RUSC. Universities and Knowledge Society Journal, 8, núm.2, pp. 77-84.

. (2013). internationalisation of higher education, an introdution on the why, how and what. An Introduction to Higher Education Internationalisation, pp. 13-46.

Delors, J. (1996). La educación encierra un tesorro. Santillana Ediciones UNESCO.

Didou, S. (2005). Internacionalización y proveedores externos de educación superior en los países de América Latina y en el Caribe: Principales Problemáticas. México: Departamento de Investigaciones Educativas Centro de Investigación y de Estudios Avanzados (DIE/CINVESTAV). 


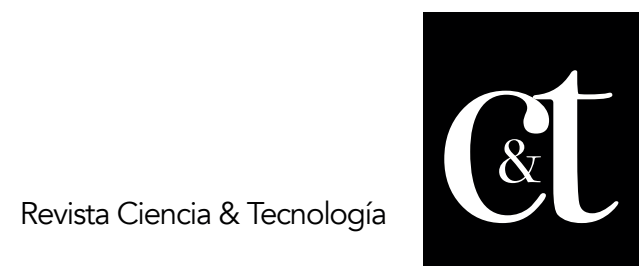

No. 29, 31 de enero de 2021

ISSN impreso: 1390 - 6321

ISSN online: 2661 - 6734

. (2017). La internacionalización de la educación superior en América Latina: transitar de lo exógeno a lo endógeno. Cuadernos de Universidades., p. 176.

Gacel-Ávila, J. (2016). Internacionalización de la educación. Diálogos del pensamiento. https://www.youtube.com/watch? $v=t W 2 A j M I A-d k$

González, E. (2011). Ciudadanía, identidades complejas y cultura política en los manuales andaluces de educación para la ciudadanía y los derechos humanos. Granada: Universidad de Granada. Retrieved from https://digibug.ugr.es/handle/10481/15724

Habermas, J. (1998). Ciudadanía e Identidad Nacional. Reflexiones sobre el futuro europeo p. 619-643. In Facticidad y Validez . Madrid: Trotta.

http://www.proyectos.cchs.csic.es/politicasmigratorias/sites/proyectos.cchs.csic.es.politicas-

migratorias/files/Ciudadania_e_identidad_nacional_-_Traduccion.pdf - (1999). La inclusión del otro. Estudios de teoía política. Barcelona: Ediciones Paidós Ibérica S.A. https://www.academia.edu/35217401/La_Inclusion_Del_Otro_Estudios_De_Te oria_Politica_Habermas_Jurgen

Heater, D. (2007). Ciudadanía una nueva historia. Madrid, España: Alianza S.A.

Hudzik, J. (2011). Comprehensive Internationalization From Concept to Action. NAFSA: Association of International Educators.

http://ecahe.eu/w/images/1/1f/Comprehensive_Internationalization__NAFSA.pdf

. (2013). Changing paradigm and practice for higher education internationalisation. An introdution to higher education. Internationalisation , pp. 45-60.

Knight, J. (1993). Internationalization: management strategies and issues, International Education, 9, pp. 6, 21-22

. (1994). Internationalization: Elements and Checkpoint. CBIE Research, 7, p. 15.

. (2003). Updated Definition of Internationalization. International higher education The Boston College Center for International Higher Education, 33, pp. 2-3.

. (2012). Student Mobility and Internationalization: trends and tribulations. Research in Comparative and International Education, 7, No. 1. https://journals.sagepub.com/doi/pdf/10.2304/rcie.2012.7.1.20

, J., \& De Wit, H. (2018). Internationalization of Higher Education: Past and Future. The Center for International Higher, 95. 


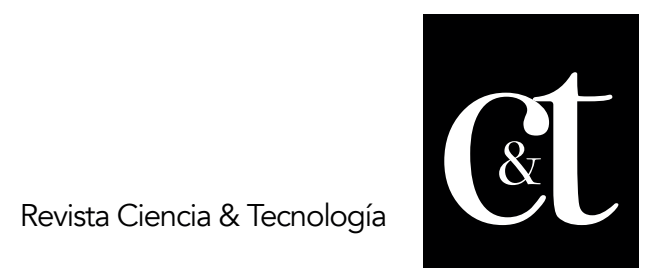

No. 29, 31 de enero de 2021

ISSN impreso: 1390 - 6321

ISSN online: 2661 - 6734

Knight, J., \& Liu, Q. (2019). International program and provider mobility in higher education: Research trends, challenges and issues. Comparative and International Education, 48, p. 18.

Knight, J., \& UNESCO. (2006). Internationalization of higher education: new directions, new challenges: 2005 IAU global survey report. http://lst-iiep.iiepunesco.org/cgi-bin/wwwi32.exe/[in=epidoc1.in] $/$ t2000 $=023277 /(100)$

Mestenhauser, J., \& Ellingboe, B. (1998). Reforming the Higher Education Curriculum: Internationalizing the Campus. Oryx Press, Phoenix, AZ.

Pedregal, R. (2003). La internacionalización de la educación superior en América del Norte ante los retos del TLCAN: un estudio comparativo. Puebla.

R. (2018). El ascendente camino de la internacionalizacion de la educación superior en China: ¿Cooperación o Competencia? México y la cuenca del pacífico, 20.

https://www.redalyc.org/jatsRepo/4337/433757506004/html/index.html\#B11

Qiang, Z. (2003). Internationalization of higher education: towards a conceptual framework. Policy Futures in Education, 2, number 2, p. 23. https://goo.gl/XpaaRw

Rudzki, R. (1998). The strategic management of intern ationalization towards a model of theoiy and practice.

Sarochar, J. (2018). Dimensiones de las nociones de ciudadanía en el primer ciclo de enseñanza secundaria en Urguay y Catañuña. Barcelona: Universitat autónoma de Barcelona.

https://ddd.uab.cat/pub/tesis/2018/hdl_10803_565885/jmsr1de1.pdf

Schuerholz-Lehr, S., Caws, C., Van Gyn, G., \& Preece, A. (2007). Internationalizing the Higher Education Curriculum: An Emerging Model for Transforming Faculty Perspectives. Canadian Journal of Higher Education, 37, No. 1, pp. 67-94. https://files.eric.ed.gov/fulltext/EJ771047.pdf

UNESCO. (1995). Educación para todos: finalidad y contexto. Conferencia mundial sobre educaci[on para todos. Jomtien, Tailandia: UNESCO. https://unesdoc.unesco.org/ark:/48223/pf0000184556

UNESCO. (2015). La educación para todos. 2000-2015: Logros y desafíos. Informe de seguimiento de la EPT en el mundo 2015.

UNESCO. (2015). Replantear la Educación. Hacia un bien común mundial? Ediciones UNESCO. https://unesdoc.unesco.org/ark:/48223/pf0000232697

Revista científica Ciencia y Tecnología Vol 21 No 29 págs. 26-41 http://cienciaytecnologia.uteg.edu.ec 


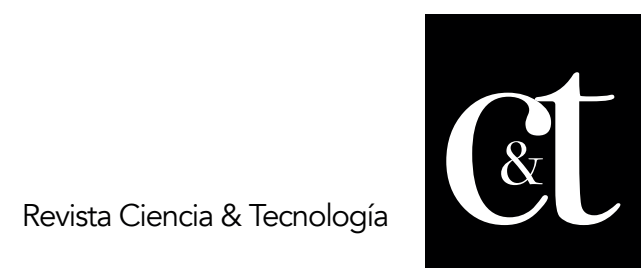

No. 29, 31 de enero de 2021

ISSN impreso: 1390 - 6321

ISSN online: 2661 - 6734

UNESCO. (2016). Objetivos de desarrollo sostenible. Agenda para el desarrollo sostenible 2030. https://www.un.org/sustainabledevelopment/es/developmentagenda/

UNESCO. (2017). La educación al servicio de los pueblos y el planeta: Creación de futuro sostenible para todos. Informe de seguimiento de la educación en el mundo 2016. Paris: UNESCO. https://es.unesco.org/gem-report/node/1279

Uribe, J. (2011). Del discurso a la realidad. Red de Universidades Públicas del Eje Cafetero colombiano: Alma Mater. Innovación Educativa, 11, núm. 56, pp. 3749. https://www.redalyc.org/pdf/1794/179421438004.pdf

Van der Wende, M. (1996). Internationalizing the Curriculum in Higher Education Report on a OECD/CERJ Stud. Tertiary Education and Management, 2, No. 2, pp. 186-195.

. (2007). Internationalization of Higher Education in the OECD Countries:

Challenges and Opportunities for the Coming Decade. Journal of Studies in International Education, 11(3-4), pp. 274-289.

doi: $10.1177 / 1028315307303543$

. (2017). Internationalisation futures in light of anti-globalisation sentiments. In M. van der Wende, A mosaic of cultures. EAIE Conference Conversation Starter (pp. 29-37). EAIE. 